\title{
Desmoplastic Non-Infantile Ganglioglioma; An Incidental Finding with Unusual Localization
}

\author{
Dimitrios Kanakis $^{1 *}$, Euripides Antoniou ${ }^{2}$ and Georgia Levidou ${ }^{3}$ \\ ${ }^{1}$ University of Nicosia Medical School, Nicosia, Cyprus \\ ${ }^{2}$ Department of Neurosurgery, Athens Medical Centre, Marousi, Greece \\ ${ }^{3}$ Department of Pathology, Klinikum Nuremberg, Paracelsus University, Nuremberg, Germany
}

*Corresponding author: Dimitrios N. Kanakis, MD, PhD, Pathologist \& Neuropathologist, Associate Professor of Pathology, University of Nicosia Medical School, St, George's, University of London Medical Programme, Delivered in Cyprus by the University of Nicosia Medical School, 21 Ilia Papakyriakou, 2414 Engomi P.O. Box 24005, CY-1700, Nicosia, Cyprus

Submission: August 07, 2017; Published: August 22, 2017

\begin{abstract}
Desmoplastic non-infantile ganglioglioma (DNIG) is a rare tumor entity and only a small number of cases have been published until now in the literature. Although its infantile counterpart (desmoplastic infantile ganglioglioma [DIG]) has been already recognized and included together with desmoplastic infantile astrocytoma (DIA) in the WHO Classification of CNS tumors, DNIG has not been accepted as a distinct tumor entity. The reason for this is obviously the scarcity of the neoplasm and subsequently the absence of substantial information regarding its pathogenesis and its association to the infantile form.
\end{abstract}

Herein we present an interesting case with an unremarkable clinical presentation, which came to medical attention after the performance of imaging investigations, for staging purposes of a multifocal papillary thyroid carcinoma. Although the tumor showed the typical biphasic nature, consisting of a solid and a cystic component, its localization in the left ventricular trigonum, was indeed uncommon. Finally, both the histomorphological and the immunohistochemical findings were consistent with the diagnosis of a desmoplastic non-infantile gangioglioma.

The occurrence of DNIG with the various clinical manifestations as well as the diverse imaging features, underlines the need of histopathological examination, in order to establish the final diagnosis.

Keywords: Desmoplastic; Non-infantile ganglioglioma

\section{Case Report}

The term desmoplastic infantile astrocytoma/ganglioglioma (DIA/DIG) was first introduced by VandenBerg et al. [1] in order to designate a distinct brain tumor, characterized by an early occurrence (usually within the first 4 months of life), voluminous size, intense desmoplasia, divergent astrocytic and ganglionic differentiation and a favorable postoperative course [1]. Although these tumors were thought initially to appear only in infants, after the first publication by Kuchelmeister et al. in 1993, who presented two such neoplasms in older individuals (15 and 25 years of age), it became obvious that they could also arise in other age groups [2]. Therefore, the name desmoplastic non-infantile ganglioglioma (DNIG) has been used to encompass these cases and since then a total of 25 cases has been published [3-8].

The typical presentation of DIG is that of a lesion which in CT examination shows, on the one hand a large hypodense or slightly hyperdense superficial solid component, with involvement of the meninges and contrast enhancement. On the other hand underneath this solid component there is also a cystic portion, which extends into the deep brain parenchyma. The MRI investigation demonstrates also these two different components, namely a large cystic (T1-hypointense, T2-hyperintense) and a smaller solid (T1-, T2-isointense) one; the latter with cortical location and leptomeningeal involvement, with homogeneous enhancement along the dura [3].

The characteristic clinical presentation of DNIGs, as previously described, involves in the majority of the cases seizures, followed by headaches and other less common symptoms [3-6]. The lesions are mainly localized within the temporal lobes followed by parietal and frontal and to a lesser extent within the occipital lobes [3].

We report here a case of a desmoplastic non-infantile ganglioglioma (DNIG) in a 35 year old woman. The tumor was identified incidentally during the staging procedure for a multifocal papillary thyroid carcinoma. In the recent medical history of the patient there were no neurological signs and symptoms. Apart from the absence of a definite clinical picture, even the localization of the present tumor is considered uncommon. The performed MRI 
showed a neoplasm measuring $1.4 \mathrm{~cm}$ in its maximal diameter, which was almost adherent to the choroid plexus of the left ventricular trigonum. The tumor consisted of both a solid and a cystic component, with the former showing contrast enhancement after gadolinium administration (Figure 1). Subsequently, during the neurosurgical procedure, the tumor was carefully detached from the choroid plexus and completely excised; a fact that was confirmed by post-surgical computed tomography.

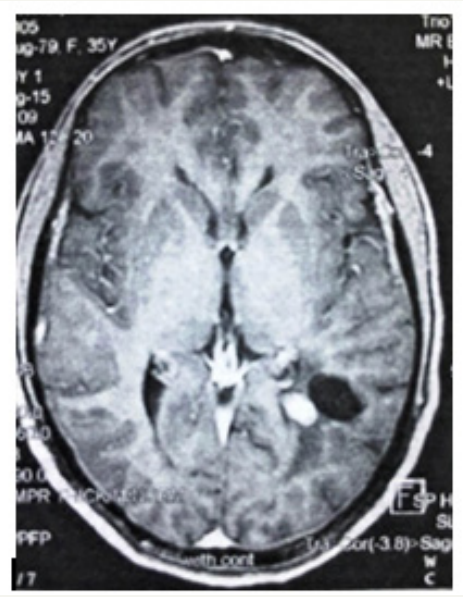

Figure 1: A tumor mass composed of a solid and a cystic component, localized to the left ventricular trigonum. Contrast enhancement of the solid portion after gadolinium administration

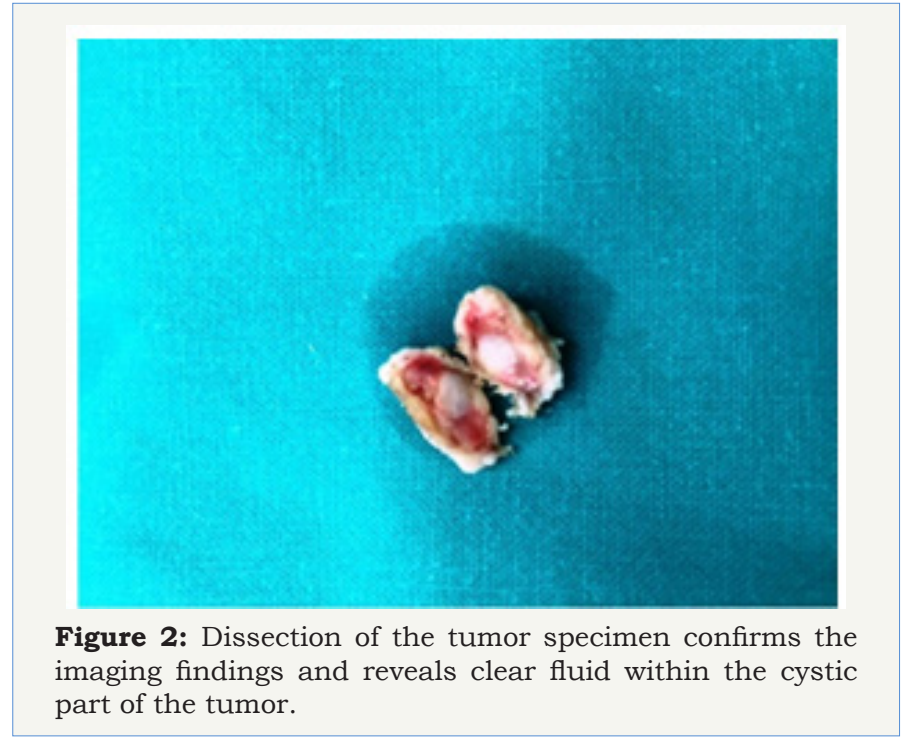

The macroscopic evaluation of the obtained specimen confirmed the biphasic nature of the lesion, but demonstrated also the presence of clear fluid within its cystic portion (Figure 2). The histopathological examination revealed a relatively circumscribed, mixed glioneuronal tumor consisting mostly of ovoid to spindleshaped cells with hyperchromatic nucleus and occasionally a prominent nucleolus as well as an eosinophilic cytoplasm (Figure 3a). These cells were arranged in a diffuse pattern, they focally

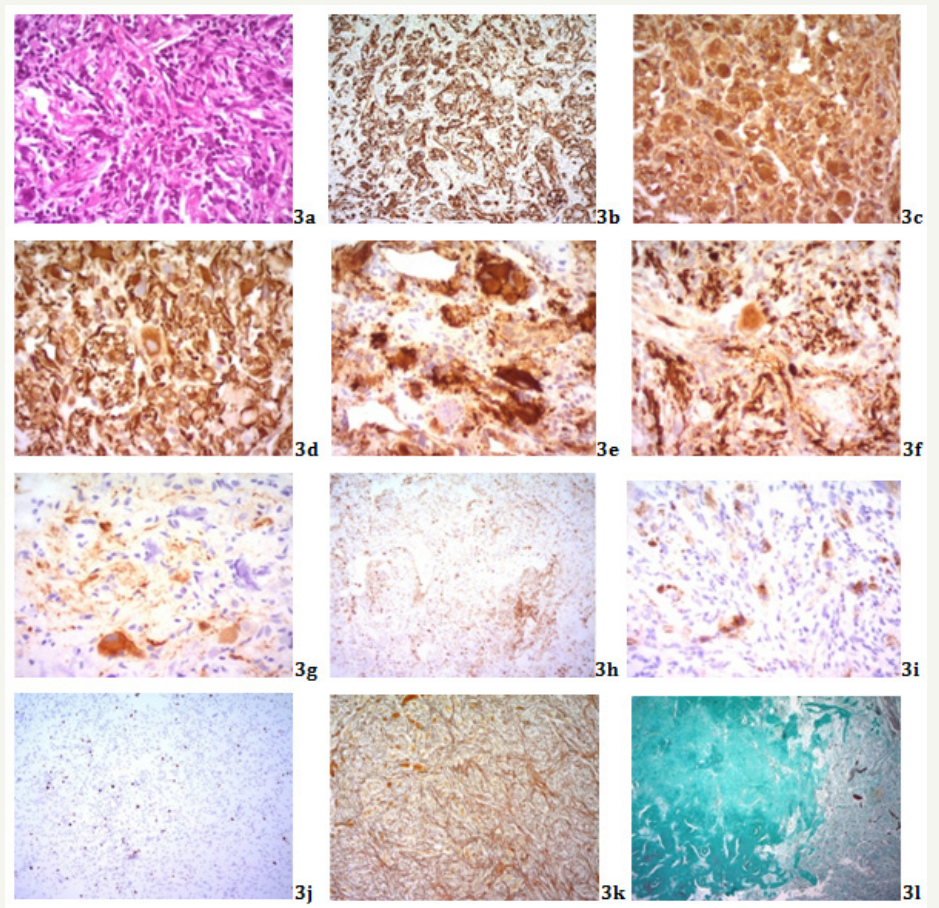

Figure 3a: Tumor consisted mainly by ovoid to spindle-shaped glial cells with hyperchromatic nuclei and eosinophilic cytoplasm. A small number of ganglionic cells and intermingled populations of round-shaped undifferentiated cells and chronic inflammatory cell infiltrates were also identifiable. Foci of calcifications were also evident. (H\&E X400). Glial cells and undifferentiated cellular elements positive for; 3b: GFAP (GFAP; X200), 3c: S100 (S100; X400) and 3d: Vimentin (VIM; X400).Ganglionic/Neuronal cells positive for: 3e: Synaptophysin (SYN; X400), 3f: Neurofilament (NF; X400) and 3g: Neuron Specific Enolase (NSE; X400), 3h: Disseminated inflammatory cell infiltrates within the tumor (LCA; X100) 3i: Remnants of meningothelial elements within the choroid plexus stained positive for Epithelial Membrane Antigen (EMA; X400). 3j: Ki67/MiB1 nuclear staining (X100). 3k: Wellformed Reticulin network (Gomori; X200).31. Dense collagen tissue within the tumor (Masson; X40). 
acquired a whirling appearance and were positive for GFAP, a finding supporting their glial differentiation, (Figure 3b), S100 (Figure 3c) and Vimentin (Figure $3 \mathrm{~d}$ ). In between them, ganglionic/neuronal cells, of variable size and occasionally plentiful cytoplasm could also be observed. These were positive for Synaptophysin (Figure 3e), Neurofilament (Figure 3f) and NSE (Figure 3g). Furthermore, a population of small, round-shaped, undifferentiated cells (Vimentin [+]; Figure 3d) but LCA [-]; Figure 3h) with little cytoplasm was intermingled among the ganglionic and glial elements whereas a smaller amount of lymphocytes/plasmacytoid cells (LCA [+]; Figure 3h) was also identifiable. Immunohistochemistry for EMA revealed also a few positive cells (probably remnants of the meningothelial elements in the stroma of the choroid plexus; Figure 3i), whereas the proliferation index through Ki67/MiB1 was low, with a nuclear staining in about 3\% of the neoplastic cells (Figure 3j). However, the most striking histological feature was the presence of a marked desmoplastic element (well-formed Reticulin network; Gomori [+]; Figure 3k), with focal development of dense collagen tissue (Masson [+]; Figure 3l). Frequent calcifications were noted throughout the tumor tissue (Figure 3a), whereas mitotic Figures were inconspicuous and tumor necrosis was not evident.

The combination of the aforementioned imaging findings with the cyto-/histomorphological and immunohistochemical findings are consistent with/lead to the diagnosis of a desmoplastic noninfantile ganglioglioma. The current case seems to have both typical and atypical features compared to the previously reported cases of DNIG. The typical ones include the biphasic solid and cystic component of the tumor, the contrast enhancement of its solid portion and the characteristic histomorphological and immunohistochemical findings. In the atypical features belongs the small size of the tumor (compared to the majority of the previously reported DNIG cases), a fact that could also explain the absence of clinical presentation. The central localization of the tumor, namely away from the meninges, as well as its adherence to the choroid plexus of the ventricular trigonum, though uncommon, have been previously reported. Similarly, the case presented by Derinkuyu et al. was localized in the parahippocampal region, adjacent to the ambient cistern and had no contact to the meninges [8]. Besides, herein as in our case, the solid component of the tumor was medial to its cystic portion. Likewise, two more cases have been described by two different groups, in which the lesions were also centrally localized and more precisely in the left peritrigonal region, with the only difference to ours being the absence of a cystic component in both instances $[9,10]$.

With regards to all the previously published DNIGs studies, we could conclude that these tumors should always be considered in the differential diagnosis, whenever an intracerebral neoplasm is evaluated, since their presentation can vary considerably not only concerning the clinical symptomatology (seizures, headaches, weakness of the extremities, etc.) but also the wide range of imaging features (central or peripheral localization, attachment or not to the meninges, mono-/ or biphasic nature). Therefore, we shall always keep in mind that the histopathological evaluation is the gold standard for the diagnosis of these rare tumor entities.

\section{Acknowledgement}

DK and GL examined the surgical specimen and interpreted both the histochemical and immunohistochemical results. DK, GL and EA prepared the manuscript. EA provided the macroscopic, whereas DK and GL the microscopic Figures. All authors approved the submitted final version of the manuscript.

\section{Ethical Approval}

The patient has given her informed consent.

\section{Conflict of Interest}

The authors declare they have no conflict of interest.

\section{References}

1. VandenBerg SR, May EE, Rubinstein LJ, Herman MM, Perentes E, et al. (1987) Desmoplastic supratentorial neuroepithelial tumors of infancy with divergent differentiation potential ("desmoplastic infantile gangliogliomas"). J Neurosurg 66(1): 58-71.

2. Kuchelmeister K, Bergman M, von Wild K, Hochreuther D, Busch G, et al. (1993) Desmoplastic ganglioglioma: report of two non-infantile cases. Acta Neuropathol 85(2): 199-204.

3. Gelabert-Gonzalez M, Serramito-Garcia R, Arcos-Algaba A (2011) Desmoplastic infantile and non-infantile ganglioglioma. Review of the literature. Neurosurg Rev 34(2): 151-158.

4. Rangel-Castilla L, Kew Y, Powell S, Zhang YJ (2011) Desmoplastic noninfantile ganglioglioma in late adulthood. J Neurooncol 103(3): 733-737.

5. Romero FR, Listik S, Fabris PA (2011) Desmoplastic ganglioglioma: report of a non-infantile case. Arq Neuro-Psiquiatr 69(2): 258-259.

6. Costa S, Marques J, Pereira P, Firmo C, Pimentel J (2012) 23-year old man with a superficial cortical brain tumor. Brain Pathol 22(3): 429-432.

7. Gundamaneni SK, Ganesh CV, Mahadevan A, Madhugiri VS, Sasidharan GM, et al. (2013) Non-infantile desmoplastic cerebellar ganglioglioma in a patient with multiple congenital anomalies: a rare association. Pediatr Neurosurg 49(2): 105-109.

8. Derinkuyu BE, Ucar M, Borcek AO, Damar C, Oztunali C, et al. (2015) Non-infantile variant of desmoplastic ganglioglioma: Conventional and advanced MR imaging characteristics. Neuroradiol J 28(3): 259-263.

9. Ganesan K, Desai S, Udwadia-Hegde A (2006) Non-infantile variant of desmoplastic ganglioglioma: a report of 2 cases. Pediatr Radiol 36(6): 541-545.

10. Khubchandani SR, Chitale AR, Doshi PK (2009) Desmoplastic noninfantile ganglioglioma: A low-grade tumor, report of two patients. Neurol India 57(6): 796-799. 